Revista Arbitrada Interdisciplinaria KOINONIA

Año VI. Vol VI. N³. Edición Especial: Educación II. 2021

Hecho el depósito de Ley: FA2016000010 ISSN: $2542-3088$

FUNDACIÓN KOINONIA (F.K). Santa Ana de Coro. Venezuela.

Estefanía Belén Silva-Flores; Luis Bolívar Cabrera-Berrezueta

http://dx.doi.org/10.35381/r.k.v6i3.1305

\title{
Aprendizaje basado en la interacción entre pares de básica elemental durante el COVID-19
}

Learning based on the interaction between pairs of basic elementary during COVID-19

\author{
Estefanía Belén Silva-Flores \\ estefania.silva.59@est.ucacue.edu.ec \\ Universidad Católica de Cuenca, Azogues \\ Ecuador \\ https://orcid.org/0000-0003-0123-7432 \\ Luís Bolívar Cabrera-Berrezueta \\ bolivarcabrera@ucacue.edu.ec \\ Universidad Católica de Cuenca, Cuenca \\ Ecuador \\ https://orcid.org/0000-0002-6853-635X
}

Recepción: 10 de abril 2021

Revisado: 05 de mayo 2021

Aprobación: 30 de junio 2021

Publicación: 15 de julio 2021 
Revista Arbitrada Interdisciplinaria KOINONIA

Año VI. Vol VI. N³. Edición Especial: Educación II. 2021

Hecho el depósito de Ley: FA2016000010

ISSN: 2542-3088

FUNDACIÓN KOINONIA (F.K). Santa Ana de Coro. Venezuela.

\title{
RESUMEN
}

La pandemia por COVID19 obligó al sistema educativo a migrar de un espacio físico específico a uno virtual ambiguo, diferente para cada niño; el aprendizaje significativo entre pares se ve marcado por el distanciamiento social, no hay convivencia. El ser humano tiene una disposición para aprender en contacto e intercambio con el otro. El propósito es conocer la percepción que tienen los tutores de E.B.E. de la U.E. "Abelardo Tamariz Crespo" frente al aprendizaje significativo entre grupo de pares en tiempos de COVID 2019. Se realizó una investigación con enfoque cualitativo etnometodológico, resaltando el rol actual de pares para alcanzar un aprendizaje significativo, donde limitantes y, oportunidades han surgido al mismo tiempo, resultando complejo poder generar la apertura de espacios de interacción; se propone una metodología que saldría de las Técnicas de Empoderamiento y Participación como lo es el Aprendizaje Basado en la Interacción entre Pares (A.B.I.P).

Descriptores: Aprendizaje activo; método de aprendizaje; aprendizaje en línea. (Palabras tomadas del Tesauro UNESCO).

\begin{abstract}
The COVID19 pandemic forced the educational system to migrate from a specific physical space to an ambiguous virtual one, different for each child; meaningful peer learning is marked by social distancing, there is no coexistence. The human being has a disposition to learn in contact and exchange with the other. The purpose is to know the perception that tutors have of E.B.E. of the U.E. "Abelardo Tamariz Crespo" in the face of significant learning among peer groups in times of COVID 2019. An investigation with a qualitative ethnomethodological approach was carried out, highlighting the current role of peers to achieve meaningful learning, where limitations and opportunities have arisen at the same time, being complex to be able to generate the opening of spaces of interaction; A methodology is proposed that would come out of the Empowerment and Participation Techniques such as Learning Based on Peer Interaction (A.B.I.P).
\end{abstract}

Descriptors: Activity learning; learning methods; electronic learning. (Words taken from the UNESCO Thesaurus). 
Revista Arbitrada Interdisciplinaria KOINONIA

Año VI. Vol VI. N³. Edición Especial: Educación II. 2021

Hecho el depósito de Ley: FA2016000010 ISSN: 2542-3088

FUNDACIÓN KOINONIA (F.K). Santa Ana de Coro. Venezuela.

\section{INTRODUCCIÓN}

Debido a la realidad que atraviesa el mundo entero a causa del coronavirus y la declaración de él Director General de la Organización Mundial de la Salud, Dr. Tedros Adhanom Ghebreyesus el 11 de marzo de 2020 al anunciar la enfermedad del COVID 2019 como una pandemia , las personas han tenido que adaptarse a un escenario totalmente distinto de lo acostumbrado, las diferentes áreas en donde se desarrolla el ser humano también se vieron afectadas, por lo que la educación al ser una de ellas se vio obligada a migrar del salón de clases a una pantalla, a convertir una presencialidad a una virtualidad; pero no es un fenómeno aislado ya que el vínculo social entre personas se transformó, ya que se encuentra bien limitada al régimen de mantener un distanciamiento social.

A nivel mundial, atravesamos una crisis en todas las dimensiones del ser humano, biopsicosocial, la cual la mayoría de países desarrollados han sabido sobrellevarla en el plano de la educación de manera óptima, ya que se cuenta con los recursos de conectividad, accesibilidad, capacitación y preparación para poder ejercer la docencia ante esta nueva modalidad; pero la situación es diferente en países tercermundistas ya que la calidad de vida marca una notable diferencia al hablar de conectividad, accesibilidad y preparación.

Es así que a nivel mundial nadie estuvo preparado para afrontar una pandemia de éste tipo, pero algunos países tuvieron una mejor respuesta que otros, como sucede en el caso de Ecuador en donde las estrategias y herramientas a nivel de los docentes resultaron escazas, hasta cierto punto nulas; ya que el ser un excelente docente en una modalidad presencial no garantiza que suceda lo mismo al estar de intermediario una pantalla la dinámica resulta desafiante en este punto ya que se pone en juego otras variantes que en lo presencial no influían directamente; como lo es la conectividad, accesibilidad, relaciones interpersonales, tiempo, metodología, herramientas, entre otros. 
Considerando los artículos 26 y 27 de la Constitución de la República del Ecuador prescriben que la educación es un derecho de las personas y un deber ineludible e inexcusable del Estado, que constituye un área prioritaria de la política pública, garantía de la igualdad e inclusión social y condición indispensable para el Buen Vivir. Las personas, la familia y la sociedad tienen el derecho y la responsabilidad de participar en el proceso educativo; Que, el artículo 44 de la Norma Constitucional prevé:

El Estado, la sociedad y la familia promoverán de forma prioritaria el desarrollo integral de las niñas, niños y adolescentes, y asegurarán el ejercicio pleno de sus derechos; se atenderá al principio de su interés superior y sus derechos prevalecerán sobre los de las demás personas.

Por otra parte, el artículo 14 de la ley orgánica de educación intercultural (LOEI) determina: "En ejercicio de su corresponsabilidad, el Estado, en todos sus niveles, adoptará las medidas que sean necesarias para la plena vigencia, ejercicio efectivo, garantía, protección, exigibilidad y justiciabilidad del derecho a la educación de niños, niñas y adolescentes". Basándose en los artículos mencionados mediante Decreto Ejecutivo No. 1017 de 16 de marzo de 2020, el Presidente Constitucional de la República declaró "el estado de excepción por calamidad pública en todo el territorio nacional, por los casos de coronavirus confirmados y la declaratoria de pandemia de COVID-19 por parte de la Organización Mundial de la Salud"; por lo que se da la Resolución el 2 de abril de 2020.

En este sentido, el Comité de Operaciones de Emergencia Nacional resolvió en cuanto al aspecto educativo: Mantener la suspensión de clases presenciales durante todo el mes de abril para todo el Sistema Nacional de Educación Escolar en todos los niveles, el cuerpo administrativo y docente del Sistema Nacional de Educación, continuará laborando mediante la modalidad de teletrabajo desde sus hogares y no deberá asistir a sus lugares de trabajo. Además, los docentes realizarán planificaciones curriculares, capacitaciones y otras actividades a su cargo en línea. 
Revista Arbitrada Interdisciplinaria KOINONIA

Año VI. Vol VI. N³. Edición Especial: Educación II. 2021

Hecho el depósito de Ley: FA2016000010

ISSN: 2542-3088

FUNDACIÓN KOINONIA (F.K). Santa Ana de Coro. Venezuela.

Debido a la pandemia del COVID 2019, la educación se vió en la necesidad de adaptarse a la nueva realidad tanto para docentes, estudiantes y sus familias, en la que la cercanía con el otro para entablar relaciones se quebró, es así que se perdió el contacto relacional con las personas limitándose a una pantalla como intermediario; afectando el aprendizaje activo entre los grupos de pares, además el tiempo limitado y reducido resalta cada vez más estas limitantes de la virtualidad como la interrelacionalidad deficiente, falta de afectividad entre docente y grupo de pares lo que impide crear un verdadero aprendizaje significativo.

El objetivo de investigación es identificar las percepciones que tienen los docentes tutores del nivel de básica elemental de la Unidad Educativa "Abelardo Tamariz Crespo" frente al aprendizaje significativo entre grupo de pares en tiempos de COVID 2019.

\section{Referencial teórico}

La presente investigación pretende, conocer e identificar las diferentes manifestaciones de la adquisición del conocimiento a través del aprendizaje significativo entre grupo de pares percibidos desde los docentes tutores del nivel de básica elemental de la Unidad Educativa "Abelardo Tamariz", describiéndolo como un proceso, el cual se ve interrumpido por el COVID 2019, en donde el factor relacional es el que se ha visto más afectado ya que hemos pasado de la presencialidad, del contacto a diario con las personas a pasar a una relación virtual, relaciones separadas por una pantalla y limitadas a un tiempo corto en donde se ha perdido el contacto con las personas que nos rodean. Concordando con fomentar el funcionamiento de grupos de aprendizaje entre pares, ya que es más factible y efectiva la actualización pedagógica, debido a que en ella está el saber enseñar, visto como un saber vivencial que no necesariamente está en manos del saber erudito del mundo académico, sino de la convivencia y actividad diaria entre estudiantes-estudiantes; como lo señala (Cerda \& López, 2011).

El ser humano nace en una sociedad, en la cual el conocimiento es una herramienta que le permite la interacción con las demás personas, y a partir de esa socialización, el sujeto 
Revista Arbitrada Interdisciplinaria KOINONIA

Año VI. Vol VI. N³. Edición Especial: Educación II. 2021

Hecho el depósito de Ley: FA2016000010

ISSN: 2542-3088

FUNDACIÓN KOINONIA (F.K). Santa Ana de Coro. Venezuela.

es capaz de relacionar y reelaborar su propio saber con otros conceptos y experiencias, es de esta forma que el ser humano es inherentemente social. De acuerdo con lo anterior, (Roig-Zamora \& Araya-Ramírez, 2014), indican que la construcción del conocimiento es una actividad tanto individual como social, en donde las interacciones docente-estudiante y estudiante-estudiante se transforman en elementos necesarios para enlazar el aprendizaje con situaciones del entorno que, a su vez, permiten la ampliación, profundización y realimentación de los conocimientos aprendidos. En este sentido, las diversas representaciones que hacen los estudiantes sobre el conocimiento son influidos por el entorno social, los estímulos y las diversas formas de aplicación.

Por otra parte, (Dosman \& Gimeno, 2010), señalan que la conversación o discusión entre pares debe ser el centro de una clase en línea, no solo porque permite sistematizar parte del contenido, sino porque crea sentido de "presencia" en un entorno tecnológico. Sin duda alguna, la conexión social es propia de las clases presenciales; no obstante, en clases virtuales este sentido se puede reforzar en los foros, ya que, a pesar de ser una actividad asincrónica, es un escenario que proporciona el contacto con los otros: aprendemos como seres sociales y en contextos sociales específicos.

Desde otra perspectiva (Mendoza-Castillo, 2020), manifiesta que la educación presencial y educación a distancia no son lo mismo, si se cree que son iguales por tratarse de educación, es una equivocación. Depende de cómo se edifica el evento educativo en cada una; existen elementos propios de la educación a distancia, que la hacen distinta de la educación presencial, debido a que repercuten en el actuar docente, el aprendizaje del alumno y la adquisición de los objetivos programáticos e institucionales. Resultando una diferencia clara el uso de la tecnología como un mediador de la educación a distancia.

Por lo tanto, los niños se ven afectados, porque dejan de asistir a clases y se cambia la forma de enseñanza que estaban acostumbrados y guiados a realizar todos los días, además de no tener una persona que les pueda guiar todo el tiempo como su maestra, ya que los padres también cumplen con otras responsabilidades. Así mismo, los niños 
Revista Arbitrada Interdisciplinaria KOINONIA

Año VI. Vol VI. N³. Edición Especial: Educación II. 2021

Hecho el depósito de Ley: FA2016000010 ISSN: 2542-3088

FUNDACIÓN KOINONIA (F.K). Santa Ana de Coro. Venezuela.

han dejado de ver a sus profesores y amigos esto ha generado una crisis educativa y emocional. "El efecto emocional, físico y psicológico derivado de la pandemia impacta de manera específica en los menores, que se enfrentan también a una situación extraña y desconocida" (Borraz, 2020).

\section{Principios de la escuela activa y Aprendizaje entre grupo de pares}

El estudiante es el centro, el eje sobre quien gira toda la enseñanza, siendo esencial que el alumno descubra el conocimiento y sus explicaciones a partir de la experiencia entre pares en donde el docente cumple un papel secundario de guía del aprendizaje como lo señala (Ruiz, 2013). En la educación tradicional sólo el docente es el encargado de transmitir los conocimientos y habilidades para generar el desarrollo intelectual de sus alumnos, se cree que el profesor es el único agente formativo en el proceso educativo. El proceso de enseñanza aprendizaje se estima así de una manera unidireccional, centrada en la relación académica entre maestro-alumno. Sin embargo, desde una educación alternativa el proceso de enseñanza-aprendizaje es un proceso complejo, donde la construcción del conocimiento y el dominio de habilidades para lograr el desarrollo integral de las personas exige la importancia del grupo de iguales en el aula como uno de los elementos más relevantes, junto con el maestro, en la elaboración del conocimiento científico, pero especialmente como formador de competencias personales y sociales, como lo indican (Berra-Bortolotti \& Dueñas-Fernández, 2008).

\section{Aprendizaje significativo}

El trabajo en grupo cumple con la resolución de problemas, en la que es importante diferentes opiniones, distintas propuestas o perspectivas de sus compañeros. Contar con varios puntos de vista ayuda a evitar errores que se relaciona con las creencias personales o con ideas fijas que puede tener un estudiante, además un alumno que se siente inseguro puede adquirir confianza en el grupo como lo indica (Viera-Torres, 2003). 
A lo que (Wenger et al. 2011), sugiere que las personas aprenden mediante la cooperación dentro de una comunidad de experiencia, en donde lo primordial es el resultado de nuevos estándares de conocimiento obtenidos por medio de la actividad e interacción en grupo, ya que la práctica siempre será una práctica social es decir un proceso de aprendizaje interactivo, sólo de esta manera existirá siempre un aprendizaje significativo.

Desde otro enfoque (De Souza, 2013), menciona tres dimensiones: el cognitivo, emocional y social; para que se produzca el aprendizaje, el cual lo simboliza como un triángulo en donde, la persona se localiza en la cúspide del mismo, el entorno social en la base, que sería el proceso externo y el proceso interno el psicológico el cual al igual que el anterior se interrelacionan e interactúan constantemente para adquirir conocimientos significativos.

\section{Aprendizaje social}

Según (Desiato, 2000), nadie se hace humano solo. Es así que sólo el contacto, la vinculación de otros seres humanos, nos convierte en humanos y por esa razón la buena educación es fabricación de humanidad. Lo que concuerda con lo propuesto por (Analuisa, 2020) que educar es el proceso formativo social del ser humano, en conocimientos y valores, direccionado a conseguir una plenitud integral.

Por otro lado, (Hartup \& Moore, 1983) mencionan la importancia de la relación entre coetáneos, a la cual no se le da énfasis en la educación; indicando que las relaciones e interrelaciones tienen un gran impacto en la adquisición de conocimientos debido a que resulta que entre niños de la misma edad se facilita el proceso de enseñanza-aprendizaje y además los niños también aprender a recibir las aportaciones y puntos de vista de los demás. 


\section{Presencialidad / Virtualidad}

La educación muta migrando a nuevos espacios que conciben de manera diferente el proceso de enseñanza-aprendizaje replanteando los roles de los actores involucrados. Por su parte el docente, que originalmente concebía como su ámbito acción las cuatro paredes del aula, desde una visión casi siempre asimétrica, se ve en la obligación de asumir que los aprendizajes deben llevarse a cabo con su presencia direccionante, mientras que en el aula todo es más predecible, en ella cada quien sabe su papel y las incertidumbres parecen haberse ido de permanentes vacaciones, como lo manifiesta (Aguilar \& Del-Valle, 2016).

\section{MÉTODO}

Luego de esbozar la base teórica, la presente investigación cuenta con los conceptos y categorías con los que se abordará esta investigación de tipo bibliográfica-exploratoriadescriptiva para la recolección de la información es necesario trazar el plan de implementación que será la guía metodológica para llegar al conocimiento de las percepciones de los docentes tutores de Básica Elemental de la Unidad Educativa "Abelardo Tamariz Crespo" frente al aprendizaje significativo entre grupo de pares en tiempos de COVID 2019.

Se ha trazado un proceso metodológico con enfoque cualitativo, no experimental y con cohorte transversal, cuya población de estudio se realizó con un muestreo de selección estratificado, con el cual se piensa alcanzar y cumplir los objetivos planteados a través de la etnometodología la cual hará hincapié en la interpretación y transformación de la realidad, centrándose en los acontecimientos cotidianos del grupo de estudio. Resultando primordial el acercamiento al grupo de estudio de manera virtual, sobre el cual se va a investigar, la cual se está obteniendo a través de la inmersión inicial, la que nos permite conocer la realidad y dinámica, generando espacios de cercanía.

En la cual se empleó una entrevista estructurada, técnica que permitió interactuar con los sujetos investigados, la cual resulta flexible para precisar conceptos y obtener mayor 
información sobre la problematización a tratar. Esta técnica, requerió el uso de instrumentos tales como un registro y una guía de preguntas, la cual requerió una validación para proceder a aplicarlas y el instrumento a utilizar serán grabaciones en audio de voz y anotaciones de campo.

Se realizó una entrevista exploratoria con los docentes tutores de Básica Elemental de la Unidad Educativa "Abelardo Tamariz Crespo", las cuales nos permitieron obtener parámetros generales sobre el problema de investigación, la entrevista aplicada fue estructurada y se realizó a los seis docentes tutores de la Básica Elemental. Después de haber realizado éste acercamiento sobre el fenómeno de estudio, se aplicó la herramienta planteada para esta investigación: seis entrevistas estructuradas, de forma individual dirigidas a los docentes tutores de Básica Elemental de la Unidad Educativa "Abelardo Tamariz Crespo". La técnica empleada permitió conocer la percepción frente al aprendizaje significativo entre grupo de pares en tiempos de COVID 2019.

Después de haber obtenido la información, procedí a su análisis e interpretación, a través de la elaboración de un marco interpretativo; para la cual se procedió a extraer de las grabaciones y notas de campo la información más relevante, la que permitió establecer la percepción de los docentes tutores, la cual después de haber ordenado, sistematizado las respuestas, se elaboró las conclusiones preliminares

Además de haber sido procesada, analizada, ubicarlas en categorías e interpretarla se elaboró el informe final, el cual nos dará un marco claro sobre las limitaciones, debilidades y recomendaciones sobre la investigación, cabe recalcar que la investigación respetó y se manejó con los siguientes aspectos éticos, tales como: anonimato y confidencialidad, responsabilidad en las evaluaciones, consideración con los informantes, socialización y convalidación de los informantes en el estudio respetando los derechos, tiempo e información; a través del consentimiento informado que se entregará a cada participante. 
Revista Arbitrada Interdisciplinaria KOINONIA

Año VI. Vol VI. N³. Edición Especial: Educación II. 2021

Hecho el depósito de Ley: FA2016000010 ISSN: 2542-3088

FUNDACIÓN KOINONIA (F.K). Santa Ana de Coro. Venezuela.

Estefanía Belén Silva-Flores; Luis Bolívar Cabrera-Berrezueta

\section{RESULTADOS}

A continuación se muestra una tabla explicativa de los resultados obtenidos de las seis entrevistas estructuradas a los 6 docentes tutores de Básica Elemental de la Unidad Educativa "Abelardo Tamariz Crespo", dividida por categorías de análisis que se obtuvo de la elaboración del marco interpretativo.

\section{Tabla 1.}

Análisis de datos cualitativos.

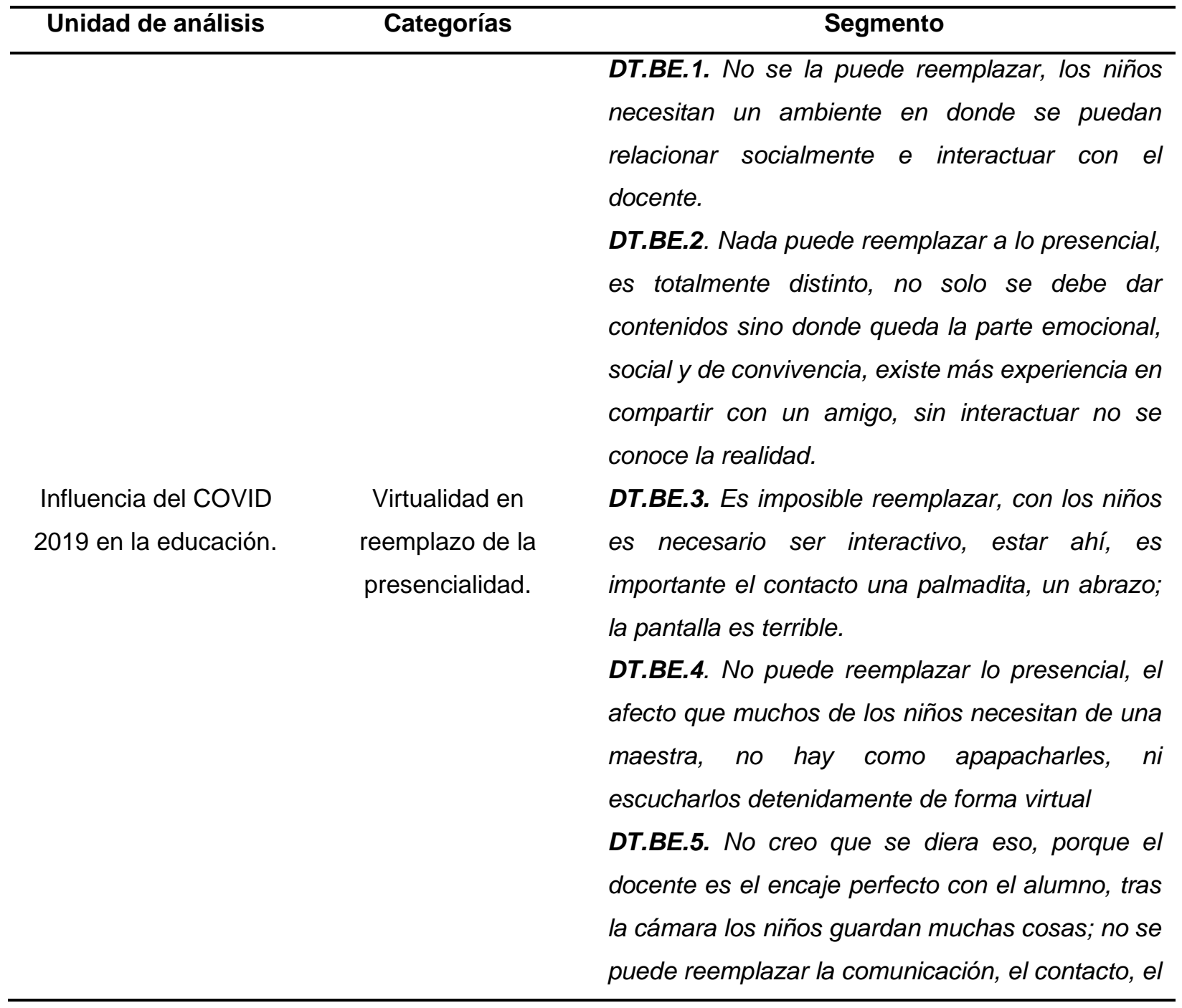


Revista Arbitrada Interdisciplinaria KOINONIA

Año VI. Vol VI. N³. Edición Especial: Educación II. 2021

Hecho el depósito de Ley: FA2016000010 ISSN: 2542-3088

FUNDACIÓN KOINONIA (F.K). Santa Ana de Coro. Venezuela.

Estefanía Belén Silva-Flores; Luis Bolívar Cabrera-Berrezueta

compartir, la emoción de estar ahí y contagiarse de ellos.

DT.BE.6. Se podría talvez en adultos, pero no en niños de primaria. No hay comparación, no se puede interactuar ni compartir.

DT.BE.1. La interacción entre pares cuenta mucho porque la relación entre ellos ayuda que se dé el aprendizaje y despejar dudas.

DT.BE.2. Permite aprender a través de las opiniones de los compañeritos.

Rol de la relación entre pares en el proceso de enseñanzaaprendizaje.
Aprendizaje significativo basado en

la interacción entre pares.
DT.BE.3. Es importante la buena relación entre ellos comprenden y solucionan problemas no solo académicos, sino emocionales también.

DT.BE.4. Como docente promovemos y dirigimos actividades para que ellos se puedan sentir bien y seguros de aprender juntos.

DT.BE.5. El aprendizaje no sólo es del docente al estudiante; entre ellos también aprenden desde sus experiencias y conocimientos, siendo muy positivo. Es así que a veces el compañero puede llegar más que el mismo docente

DT.BE.6. Es importante porque cambian opiniones, dan su punto de vista y todo ello es un gran aporte para el aprendizaje.

DT.BE.1. Ha regresado lo tradicional, por lo que el docente prácticamente es el que más está hablando

DT.BE.2. No se ha regresado, porque en lo tradicional estaba lejos de aplicarse la tecnología. Captar la atención en lo presencial es difícil no se diga ahora en la virtualidad es necesario 
Revista Arbitrada Interdisciplinaria KOINONIA

Año VI. Vol VI. N³. Edición Especial: Educación II. 2021

Hecho el depósito de Ley: FA2016000010 ISSN: 2542-3088

FUNDACIÓN KOINONIA (F.K). Santa Ana de Coro. Venezuela.

Estefanía Belén Silva-Flores; Luis Bolívar Cabrera-Berrezueta

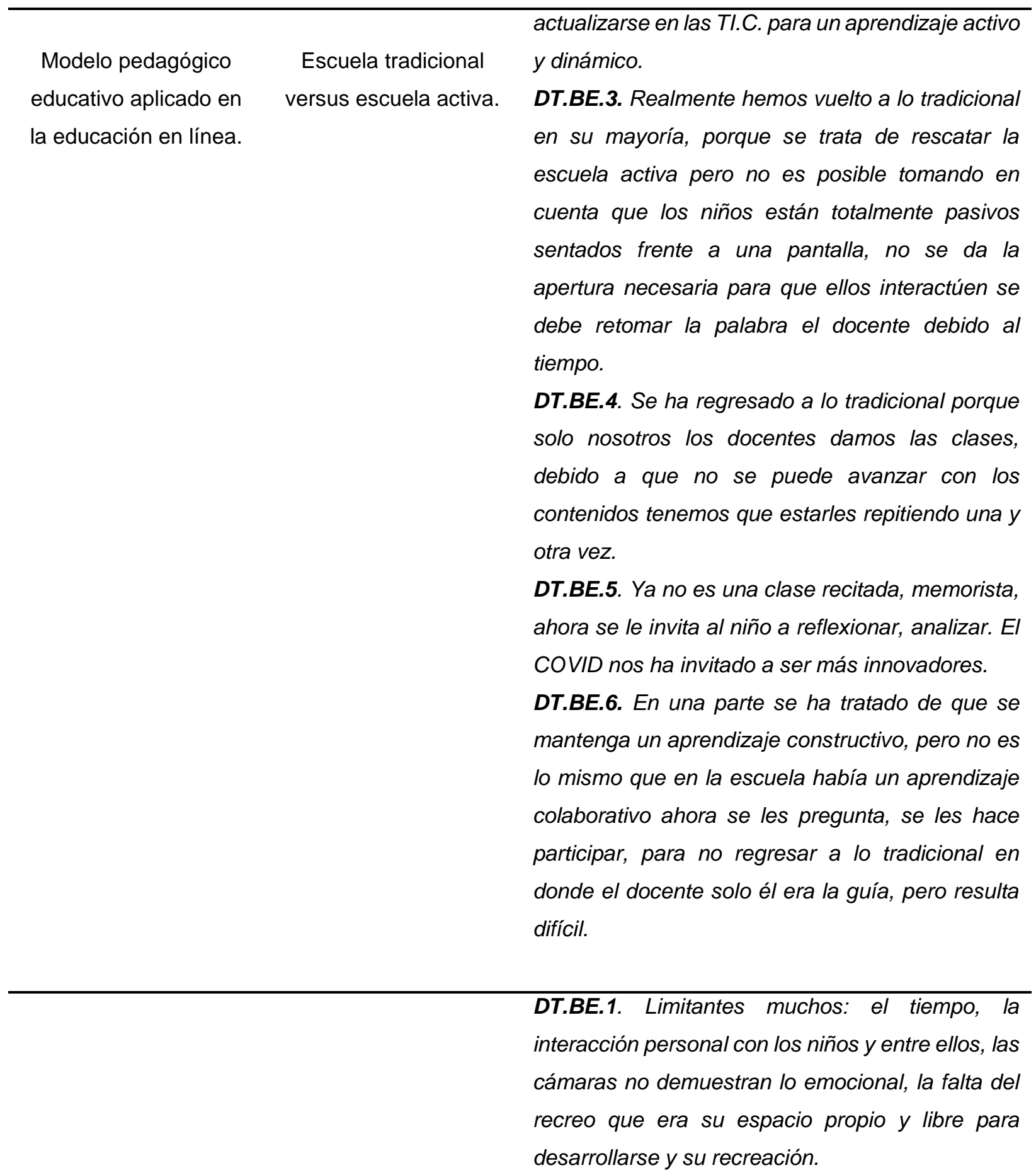


Revista Arbitrada Interdisciplinaria KOINONIA

Año VI. Vol VI. N³. Edición Especial: Educación II. 2021

Hecho el depósito de Ley: FA2016000010 ISSN: 2542-3088

FUNDACIÓN KOINONIA (F.K). Santa Ana de Coro. Venezuela.

Estefanía Belén Silva-Flores; Luis Bolívar Cabrera-Berrezueta

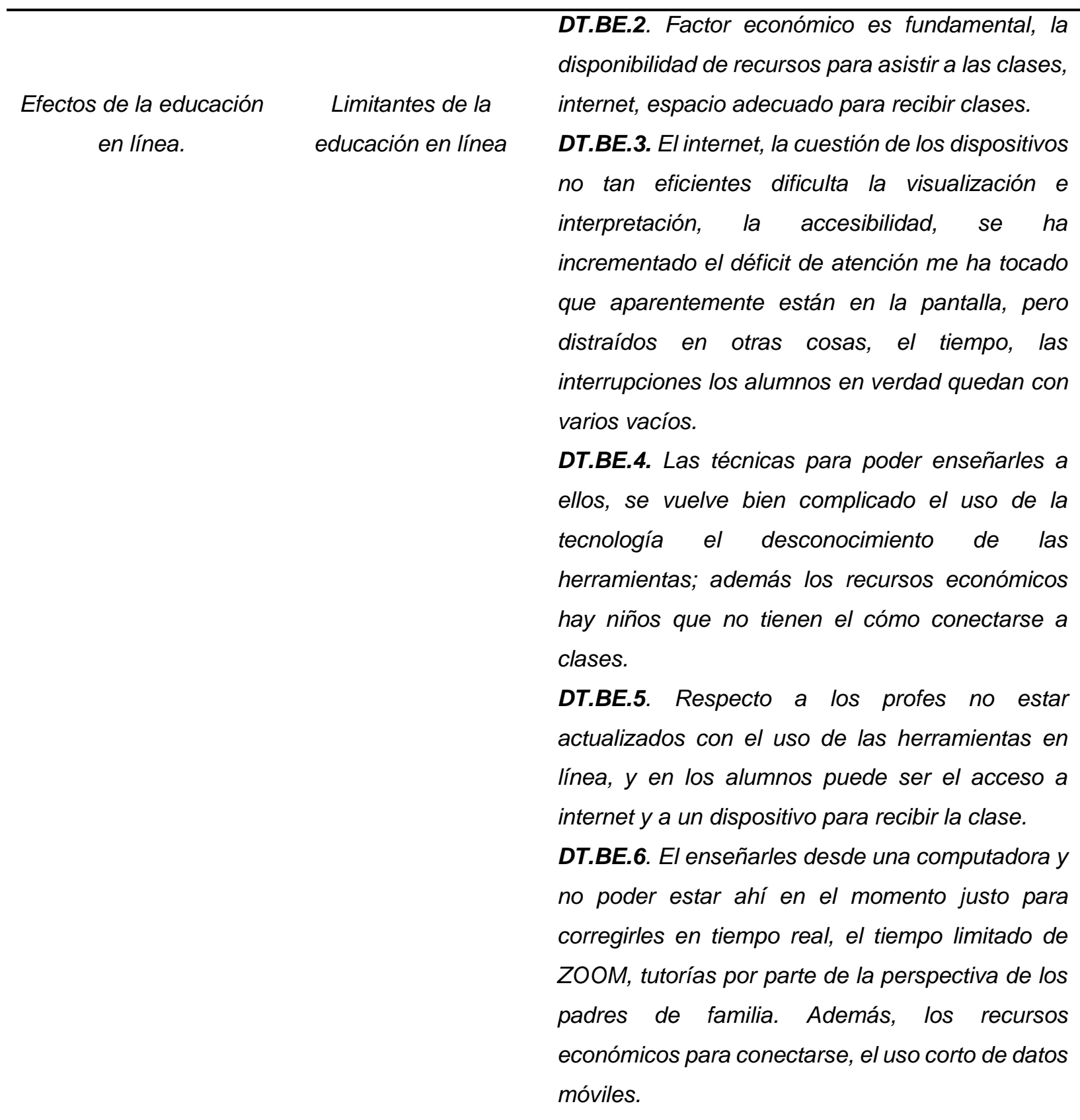

Fuente: Entrevistas. 
Revista Arbitrada Interdisciplinaria KOINONIA

Año VI. Vol VI. N³. Edición Especial: Educación II. 2021

Hecho el depósito de Ley: FA2016000010

ISSN: 2542-3088

FUNDACIÓN KOINONIA (F.K). Santa Ana de Coro. Venezuela.

En el análisis de las percepciones generadas a través de las entrevistas realizadas, se visualiza el impacto que ha tenido la pandemia del COVID 2019 en el ámbito educativo, ya que se muestra como los docentes tutores priorizan la necesidad urgente de la presencialidad en clases, según sus respuestas ven a este aspecto de la educación irremplazable ya que enseñar no solo consiste en transmitir conocimientos y contenidos, sino engloba la parte emocional y social que es inherente del ser humano, por lo que resulta importante mencionar que el individuo antes de ser razón es socialmente emocional; resultando la pantalla un lente focal que solo permite ver una minúscula parte de lo que es realmente un estudiante.

Por otra parte la relevancia de la relación entre pares es notable, ya que desde el grupo de estudio el contacto y la interacción que se mantiene entre los alumnos resulta favorable en la adquisición del aprendizaje significativo, puesto que el proceso de enseñanza-aprendizaje no es unidireccional y solo de docente a estudiantes; sino que posee varias direcciones entre una de ellas el grupo de coetáneos, el cual brinda la oportunidad de dar a conocer, recibir, contrastar, debatir opiniones e ideas del mismo tema, resultando enriquecedor en la consolidación del conocimiento; entre ellos se da una sana discución y conceso debido a la cercanía y sentido de pertenencia por compartir características semejantes, a este hecho se le suma la perdida del recreo espacio propio de los estudiantes en donde se fortalecían las relaciones de vínculo.

En cuanto al modelo pedagógico que se maneja ahora en la atualidad con relación a la Pandemia del COVID 2019, entre los docentes tutores se logra evidenciar que debido a la educación en línea ha sido dificil tratar de mantener el modelo pedagógico de la escuela activa dentro del aula virtual, debido a las preocupaciones de avanzar con los contenidos en tan poco tiempo, aseguran que hasta cierto punto se ha regresado al aspecto transmisivo y repetitivo, pero también hacen hincapie en que debido a la pandemia se pudo conocer otras formas de enseñar a través de la tecnología como el acceso a videos, canciones, multimedia que resulta innovador, novedoso para lograr captar la atención de los niños y rescatar de alguna forma la escuela activa; asimismo se 
ha generado la vinculación de manera positiva de los padres de familia que son un pilar fundamental en la actual escuela en línea.

Además se da a conocer uno de los efectos de la educación en línea, como lo son las limitantes del mismo, en donde se enfatiza en el factor económico, siendo necesario recalcar que los docentes tutores pertenecen a una Unidad Educativa Fiscal, en donde la mayoría de los niños no disponen de acceso a Internet, y si logran acceder es en alguna red pública como parques o en el mejor escenario a través de datos lo que resulta limitado encajando en términos de conectividad y accesibilidad, asimismo es preciso considerar desde el dispositivo en el que se entra a clases ya que la diferencia es clara en ingresar desde una computadora o celular de alta gama, al ingresar en un celular con las características básicas en donde la exposición del docente y visualización del material compartido resulta ineficiente para leerlo y entenderlo.

Esta limitante no se encuentra aislada ya que aparecen muchas más, como el factor relacional relativamente segado debido a las medidas de aislamiento y sana distancia en donde la relación entre pares en clases es casi nula, los docentes argumentan que se debe al tiempo limitado de dar clases y a las varias interrupciones que existen, ya que al no estar en el mismo espacio repercuten diferentes inconvenientes y distractores, como el hecho de estar visualmente al frente de la pantalla pero no necesariamente atentos a la clase ya que el espacio en donde se recibe las clases esta lleno de distractores y estímulos para el niños, a esto se suma la calidad de el ancho de banda de Internet en donde a veces se cae la red impidiendo un aprendizaje continuo.

Al no estar ahí en el momento justo en donde se equivocan, se obvian procesos de retroalimentación que son precisos para avanzar en la enseñanza,por consiguiente el hecho de ser un buen docente en lo presencial no es un indicador para serlo en lo virtual en donde las herramientas y métodos son diferentes y la actualización continua, conocimiento y manejo adecuado de los recursos resulta obligatorio e indispensable para poder crear espacios de interacción efectivos para alcanzar un aprendizaje significativo. 


\section{PROPUESTA}

A partir del estudio llevado a cabo se puede proponer el implemento del Método A.B.I.P. (Aprendizaje Basado en la Interacción entre Pares), debido que por la situación actual del COVID 2019 se ha dejado de lado este proceso el cual es fundamental para la adquisición de conocimientos.

El Aprendizaje Basado en la Interacción entre Pares es una metodología que permite a los estudiantes adquirir conocimientos y aprendizajes significativos a través de la relación e interacción con el otro, mediante el contacto con su coetáneo el alumno da su opinión e ideas y recibe de parte del grupo aportaciones y diferentes perspectivas las cuales forman en su conjunto una consolidación de la información, teniendo cada uno de ellos un rol en la participación de la elaboración de su aprendizaje y siendo el docente la guía formativa que consolidará el estudio realizado a través de un feedback. Para su aplicación es necesario seguir los siguientes pasos:

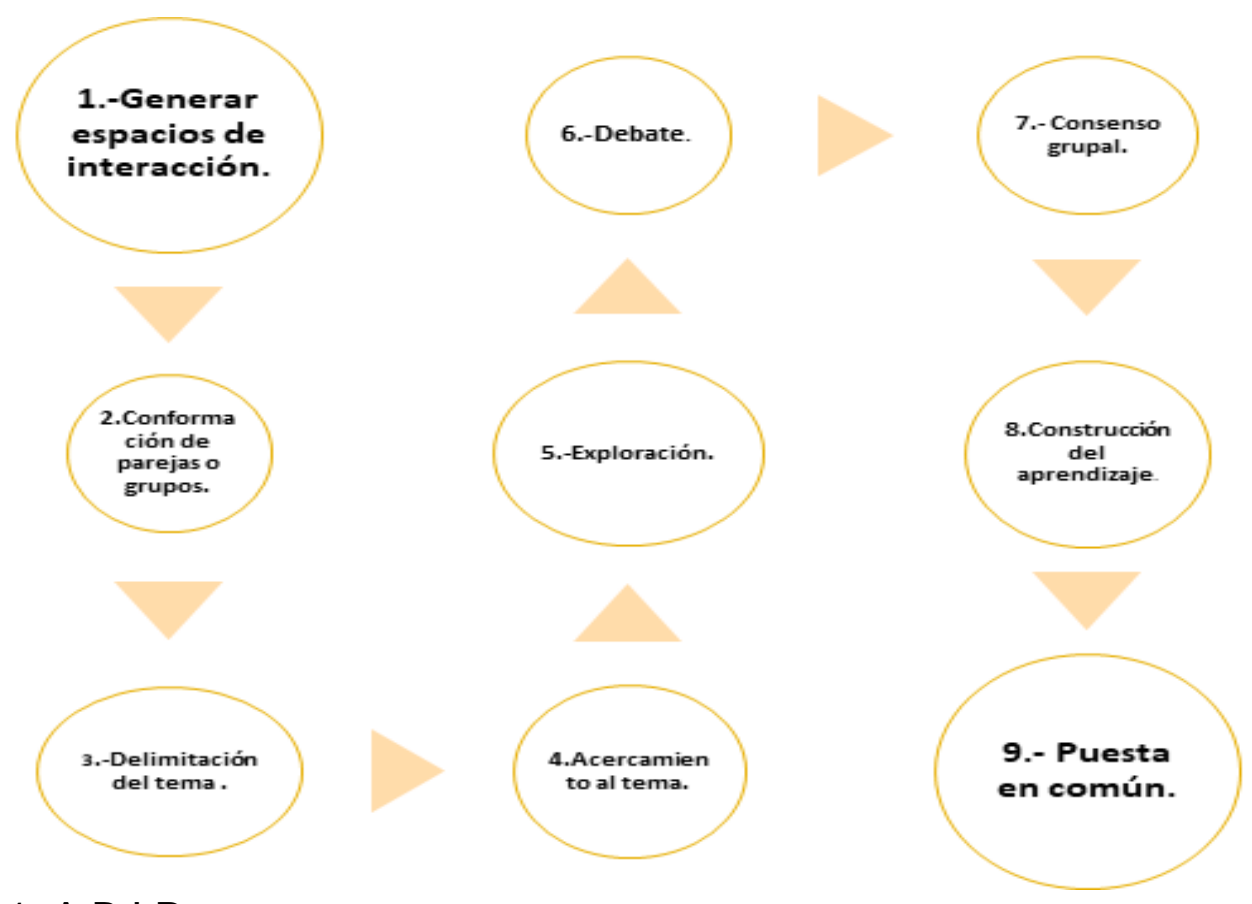

Figura 1. A.B.I.P.

Elaboración: Los autores. 
1.- Generar espacios de interacción. - Para abrir estos sitios es fundamental conocer y establecer un ambiente de rapport con los estudiantes, existiendo dos formas de incorporarse al Aprendizaje Basado en la Interacción entre Pares; la de pertenecer como lo hacemos todos al articular en algún grupo y la otra participar que se basa en la deliberación y escoger su propia y consensuada explicación.

2.-Conformacíon de parejas o grupos. - Es necesario formar parejas, tríos o máximo cuartetos; en los cuales se permite desarrollar la seguridad en ellos mismos, en lo que saben y son capaces de hacer, acrecentando sus potencialidades para pensar, sentir, hablar, crear, opinar y aprender.

En esta área se designará los roles de cada participante, como lo son:

a.-) Coordinador. - La función de este participante es distribuir las actividades propuestas para el desarrollo sistemático del A.B.I.P.

b.-) Águila. - Es el encargado de velar que se desarrollen las evidencias del proceso educativo en cada uno de los miembros del grupo, manteniéndose atento con el cumplimiento de los acuerdos básicos de los integrantes.

c.-) Crítico. - Es aquel que exhibe los diferentes desafíos y perspectivas, que se presentan en el desarrollo, afronta y presenta los riesgos de las actividades a realizarse. d.-) Coaching. - Busca conseguir la ilusión que en el encuentro afloren, las mejores capacidades, dotes y respuestas valorativas a las diferentes circunstancias que se van mostrando en el proceso.

3. Delimitación del tema. - El docente es el encargado de establecer el tema, sus contenidos y los parámetros del trabajo en donde los estudiantes podrán adquirir las habilidades necesarias para trabajar en equipo, llevar a cabo en tiempo y forma los objetivos planteados, facultando de forma eficiente la administración del bien común. 
4.- Acercamiento al tema: El grupo conformado podrá acceder a consultar revisión bibliográfica, documental, digital con la finalidad de cubrir las necesidades para el desarrollo y encontrar soluciones al tema propuesto.

5.-Exploración. - Momento en el que el grupo junta las aportaciones individuales para que se abran instancias en el que los estudiantes puedan demostrar sus respectivos criterios basados en la consulta realizada, desarrollando una argumentación sostenida.

6.-Debate. - Propiciar y provocar con seriedad una discusión pluralista en donde se abran las instancias de deliberación.

7.- Consenso Grupal. - A base de una reflexión y análisis el grupo consigue, reconoce e identifica la condición fundamental propuesta en el contenido.

8.- Construcción del aprendizaje. - Interiorización del conocimiento obtenido, el mismo que podrán hacer efectivo en los espacios requeridos, o relacionarlo con sus actividades diarias.

9.-Puesta en común. - Una vez finalizado el trabajo por parte del grupo se efectuará el discernimiento final, en el que los estudiantes manifiesten el sentir, el hacer y el pensar que les provocó en el desarrollo del tema que juntos construyeron, basándose en el descubrimiento tanto individual y colectivo como aporte único de su grupo. 
Revista Arbitrada Interdisciplinaria KOINONIA

Año VI. Vol VI. N³. Edición Especial: Educación II. 2021

Hecho el depósito de Ley: FA2016000010 ISSN: 2542-3088

FUNDACIÓN KOINONIA (F.K). Santa Ana de Coro. Venezuela.

\section{CONCLUSIONES}

La presencia de la pandemia del COVID 2019, no hay que verlo como un fenómeno temporal sino permanente, porque sin duda ha marcado las tres grandes dimensiones del ser humano como lo son: lo bio-psico-social en el cual, el ámbito de la educación tiene un antes y un después por la ganancia de nuevos métodos, técnicas y herramientas que facilitan el proceso de aprendizaje, por lo que es necesario la actualización constante en el manejo correcto de las mismas.

El impacto en el área social es uno de los más afectados, puesto que la el hombre es un ser inherentemente social desde el momento en que nace depende de alguién más para sobrevivir y el conocimiento que obtiene es a través del contacto con las redes que lo rodean, es así que la educacion fue, es y será un proceso social que humaniza.

El método de Aprendizaje Basado en la Interacción entre Pares propuesto, se refleja en la necesidad de fortalecer el aspecto relacional en el proceso de enseñanza, porque es un punto clave para la adquisición de un aprendizaje significativo, que no contaba con la apertura necesaria en la educación en línea actual.

Resulta fundamental indicar que el COVID 2019 en relación a la educación dio a conocer varias limitantes, pero al mismo tiempo generó nuevas oportunidades que nos obligaron a repensar y deconstruir la escuela, el proceso, la metodología, técnicas y herramientas para readaptarnos a las condiciones actuales de la realidad dinámica en la que estamos inmersos.

\section{FINANCIAMIENTO}

No monetario.

\section{AGRADECIMIENTO}

A la Jefatura de Posgrados de la Universidad Católica de Cuenca por permitir el desarrollo y fomento de la investigación. 


\section{REFERENCIAS CONSULTADAS}

Aguilar, M. A., \& Del-Valle, M. (2016). De lo presencial a lo virtual: Caso Universidad Metropolitana [From face-to-face to virtual: Metropolitan University case]. Opción, 32(9).

Analuisa, E. (2020). Análisis de la implicación de las familias en la educación de sus hijos/as durante la Emergencia Sanitaria generada por el Covid-19 [Analysis of the involvement of families in the education of their children during the Health Emergency generated by Covid-19]. Pontificia Universidad Católica Del Ecuador. http://repositorio.puce.edu.ec/xmlui/handle/22000/18190

Berra-Bortolotti, M., \& Dueñas-Fernández, R. (2008). El Grupo De Iguales En La Formación De Habilidades Sociales [The Peer Group In Social Skills Training]. Xihmai, 3(5), 1.

Borraz, M. (2020). Así afecta a los niños el confinamiento: pautas para gestionar la cuarentena con hijos y no tirarte de los pelos [This is how confinement affects children: guidelines for managing quarantine with children and not pulling your hair out]. https://n9.cl/hnl9

Cerda, A., \& López, I. (2011). El grupo de aprendizaje entre pares una posibilidad de favorecer el cambio de la prácticas cotidianas de aula [The peer learning group a possibility to favor the change of daily classroom practices]. Cpeip, 1-6. https://n9.cl/qo2n

Constitución de la República del Ecuador 2008. Registro Oficial 449 de 20-oct-2008 Ultima modificación: 13-jul-2011. https://n9.cl/hd0q

De Souza, L. (2013). Teorias contemporâneas da aprendizagem [Teorías contemporáneas del aprendizaje]. https://doi.org/10.19091/reced.v1i29.293

Desiato, M. (2000). Reseña de "El valor de educar" de Fernando Savater [Review of "The value of educating" by Fernando Savater]. Educere, 4(11),267-268.

Dosman, E., \& Gimeno, C. (2010). La vida y la época de Raúl Prebisch, 1901-1986 [The life and times of Raúl Prebisch, 1901-1986]. MADRID; BARCELONA; BUENOS AIRES: Marcial Pons, Ediciones Jurídicas y Sociales. doi:10.2307/j.ctv10rr97h

Hartup, W. W., \& Moore, S. G. (1990). Early peer relations: Developmental significance and prognostic implications. Early Childhood Research Quarterly, 5(1), 117. https://doi.org/10.1016/0885-2006(90)90002-I 
Revista Arbitrada Interdisciplinaria KOINONIA

Año VI. Vol VI. N³. Edición Especial: Educación II. 2021

Hecho el depósito de Ley: FA2016000010 ISSN: $2542-3088$

FUNDACIÓN KOINONIA (F.K). Santa Ana de Coro. Venezuela.

Estefanía Belén Silva-Flores; Luis Bolívar Cabrera-Berrezueta

Ley orgánica de educación intercultural LOEI. Recuperado desde https://n9.cl/go3a

Mendoza-Castillo, L. (2020). Lo que la pandemia nos enseñó sobre la educación a distancia [What the pandemic taught us about distance education]. Revista Latinoamericana de Estudios Educativos, 50(ESPECIAL), 343-352. https://doi.org/10.48102/rlee.2020.50.especial.119

Roig-Zamora, J., \& Araya-Ramírez, J. (2014). El aprendizaje entre iguales: Una experiencia didáctica para la construcción del conocimiento en la educación superior [Peer learning: A didactic experience for the construction of knowledge in higher education]. Revista Comunicación, 23(1), 17-39

Ruiz, G. (2013). La teoría de la experiencia de John Dewey: significación histórica y vigencia en el debate teórico contemporáneo [John Dewey's experience theory: historical significance and relevance at contemporary pedagogical debate]. Foro de Educación, 11(15), 103-124. https://doi.org/10.14516/fde.2013.011.015.005

Viera-Torres, T. (2003). El aprendizaje verbal significativo de Ausubel. Algunasconsideraciones desde el enfoque histórico cultural [Ausubel's significant verbal learning. Some Considerations from the Cultural Historical Approach]. Universidades, (26),37-43

Wenger, E \& Trayner, B \& Laat, M. (2011). Promoting and Assessing Value Creation in Communities and Networks: A Conceptual Framework. Ruud de Moor Centrum, 18(August), 1-60. 